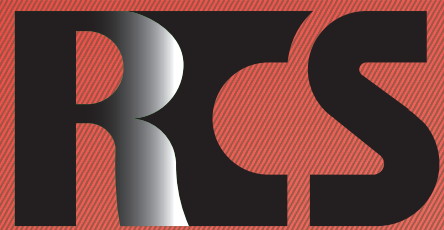

Depósito legal ppi $201502 Z U 4662$

Esta publicación científica en formato digital es continuidad de la revista impresa Depósito Legal: pp $197402 Z$ Z789

- ISSN: 1315-9518 • ISSN-E: 2477-9431

Universidad del Zulia. Revista de la Facultad de Ciencias Económicas y Sociales Vol. XXVI. No.1

\title{
Enero-Marzo 2021
}




\title{
Nueva propuesta de financiamiento público a partidos políticos en México
}

\author{
Arredondo, Verónica* \\ Vega Esparza, Reina Margarita** \\ Villegas Santillán, María Teresa*** \\ Álvarez Diez, Rubén Carlos****
}

\section{Resumen}

El financiamiento público a los partidos políticos es uno de los temas que generan polémica y discusión entre la ciudadanía, en particular en el electorado mexicano (población de estudio en este trabajo, periodo 2008 al 2020). El sistema actual de la administración mexicana, con respecto a la repartición de recursos a los partidos políticos, ignora algunos elementos importantes para alcanzar un trato equitativo entre los diversos partidos políticos, tal como la barrera a la entrada del reparto de los recursos disponibles para éstos. El objetivo central de esta investigación es proponer una metodología para estimar y distribuir el financiamiento público a los partidos políticos en México. Dicha metodología utiliza el modelo de Promedios Móviles Ponderados para estimar la Votación Total Emitida. Asimismo, se propone una nueva función para la barrera a la entrada de reparto de recursos entre partidos. Se determinó que la tasa de participación del voto es alrededor del $55 \%$, otorgándose en general, a los partidos políticos, un $45 \%$ más de los recursos que deberían de obtener; tomando en cuenta el número de votos obtenidos por cada uno de estos. Se concluyó, que se generarían ahorros por encima del $50 \%$ en el gasto del erario público.

Palabras clave: Sistemas electorales; recursos; partidos políticos; financiamiento; erario público.

Doctora en Administración. Doctora(c) en Economía. Magister en Economía. Licenciada en Matemáticas. Docente - Investigadora de la Universidad Autónoma de Zacatecas "Francisco García Salinas", México. E-mail: varredon@uaz.edu.mx (iD) ORCID: https://orcid.org/0000-0002-8475-4541

** Doctora en Metodología de la Enseñanza. Magister en Administración. Especialidad en Fiscal. Licenciada en Contaduría. Docente - Investigadora de la Universidad Autónoma de Zacatecas "Francisco García Salinas", México. E-mail: reinavega_62@yahoo.com.mx (iD) ORCID: https://orcid. org/0000-0001-5151-5977

*** Doctora en Administración Pública. Magister en Administración. Especialidad en Fiscal. Licenciada en Contaduría. Docente - Investigadora de la Universidad Autónoma de Zacatecas "Francisco García Salinas”, México. E-mail: cpmtvs@hotmail.com iD ORCID: https://orcid.org/0000-0002-6985-5256

***** Doctor en Administración Pública. Magister en Negocios Internacionales y Mercadotecnia. Licenciado en Administración. Docente - Investigador de la Universidad Autónoma de Zacatecas "Francisco García Salinas”, México. E-mail: ruben@unizacatecas.edu.mx rubenalvarezdiez@hotmail.com

ORCID: https://orcid.org/0000-0002-0877-2238

Recibido: 2020-09-09 · Aceptado: 2020-11-27 


\title{
New proposal for public financing of political parties in Mexico
}

\begin{abstract}
Public financing to political parties is one of the issues that generate controversy and discussion among citizens, specifically in the Mexican electorate (population of study in this research, period 20082020). The actual system of Mexican administration, ignores some important elements to reach an equitable treatment between the diversity of political parties, such as the threshold of resources' distribution for the political parties. The main objective of this research is to propose a methodology for the estimation and distribution public financing to Mexican political parties. The proposed methodology uses the Weighted Moving Average Model to estimate the Total Vote Cast. Additionally, it is proposed a new function to estimate the threshold of resources' distribution for political parties. It is determined that the vote cast is around 55\%, granting generally to political parties $45 \%$ more resources that they should have obtained, taking into account the number of votes earned by each of these. It is also concluded that the proposal would generate over $50 \%$ savings in the public spending.
\end{abstract}

Keywords: Electoral systems; resources; political parties; financing; public treasury.

\section{Introducción}

Actualmente, ciudadanos y diversas organizaciones sociales se han manifestado en contra del financiamiento público que reciben los partidos que integran el sistema político mexicano; o al menos han hecho visible su interés en conocer cómo se designa y se define instrumentalmente el presupuesto que se otorga a estas instituciones.

Uno de los argumentos principales que se esgrime es que el monto de esta prerrogativa debe disminuir porque es superior al que se concede a dependencias más relevantes para el desarrollo social y económico; pero, y quizá no sea tan palpable, la democracia, como bien público, requiere que el Estado encuentre o establezca las formas de financiar a los partidos políticos, puesto que son entidades de interés colectivo que promueven la competencia política y la democracia, lo cual también coadyuva en los cambios necesarios para el desarrollo integral de una sociedad. $\mathrm{Si}$ el aparato estatal instituye las normas de financiamiento de los partidos, se evita que los intereses privados, y el afán de lucro de grupos empresariales, constituyan autocracias contrarias al interés general.
Bajo este contexto de insatisfacción ciudadana, es decir, el financiamiento a los partidos políticos, resulta importante considerar que la disponibilidad de modelos para analizar las variables que determinan el adecuado monto y reparto de la subvención a los partidos - con base en distintos criterios para maximizar la equidad entre los que participan en los procesos electorales - es limitada. La situación, entonces, no garantiza la existencia de un sistema democrático moderno, pero presenta la oportunidad de entablar una discusión de donde emerja un nuevo tipo de soluciones.

La presente investigación se enfoca en la falta de un modelo de financiamiento público a los partidos políticos, desarrollado con base en la proporcionalidad entre el porcentaje de votos y el de recursos otorgados a éstos, el reparto equitativo del monto calculado, y de sus efectos en la competencia electoral.

Bajo esa perspectiva, este estudio tiene como objetivo presentar un modelo para determinar el financiamiento público que incluya las variables mencionadas y una barrera de entrada al reparto de éste; además de compararlo con el modelo vigente, para evidenciar los posibles ahorros y demostrar 
Arredondo, Verónica; Vega Esparza, Reina Margarita; Villegas Santillán, María Teresa y Álvarez Diez, Rubén Carlos

Nueva propuesta de financiamiento público a partidos politicos en México

que el financiamiento público a los partidos políticos puede reducirse en función de una nueva propuesta metodológica.

El modelo para determinar la disminución del financiamiento público a los partidos políticos se justifica porque introduce variables que modifican razonablemente el volumen de recursos monetarios y aumentan la equidad en su distribución, con resultados positivos para el desarrollo de la democracia.

En ese sentido, este artículo se compone en la primera parte, por el fundamento teórico referente a un recorrido a través del tiempo del financiamiento de partidos políticos en México; donde se definen, además, algunos de sus elementos sustanciales. En la siguiente sección se desarrolla la metodología empleada para elaborar la nueva propuesta de asignación de recursos; para ello se toma en cuenta una estimación de la Votación Total Emitida, se recurre al método de Promedios Móviles Ponderados y se propone una nueva forma de estimar la barrera a la entrada de reparto de recursos financieros. Finalmente, se presentan los resultados y conclusiones respectivas.

\section{Fundamento teórico}

\subsection{Sistemas de financiamiento de los partidos políticos}

Históricamente, se puede exponer se han constituido tres sistemas para financiar a los partidos políticos, estos son de carácter: Privado, público y mixto. De acuerdo con Zovatto (2017), el sistema de financiamiento privado, comprende las aportaciones en dinero o en especie de particulares; sin distinguir si estos son militantes o simpatizantes. El sistema público, es equivalente al concepto de financiamiento legal otorgado por el Estado y que de hecho forma parte de su mismo presupuesto; este es el que se abordará en este trabajo. Respecto al sistema de financiamiento mixto, este se estructura por el registro y aportación que ocurre como una combinación de fondos públicos y privados.
Resulta importante destacar que, existe un concepto de financiamiento más: El financiamiento político, que se refiere a la parte del dinero existente en el proceso político, y comprende "el financiamiento (legal e ilegal) de las actividades en curso de los partidos políticos y de las campañas electorales" (Ohman, 2015, p.2). El financiamiento político, de acuerdo con Zovatto (2017), resulta de la política de ingresos y egresos de los partidos políticos, y difiere del concepto de financiamiento público porque este:

Se refiere al empleo de recursos públicos en beneficio de los partidos y las campañas. Puede hacerse de manera directa, transfiriendo a los partidos dinero del presupuesto nacional, o de manera indirecta, mediante la concesión de ventajas, prerrogativas, aportes en especie o subvenciones a favor de los partidos o de las campañas electorales. (p.19)

$\mathrm{Si}$ se contextualiza lo que se dice en Ohman (2015) y Zovatto (2017), se entiende que: La subvención de los partidos es el conjunto de recursos económicos que estos obtienen de fuentes públicas y privadas, legales e ilegales, para financiar sus funciones públicas. El financiamiento político se divide en legal e ilegal. En ambos conceptos se incluye, además, la subvención privada.

Como financiamiento legal, se va a entender al conjunto de prerrogativas que el Estado autoriza a los partidos políticos, porque éste considera a las instituciones partidistas como entidades de interés público. Este tipo de financiamiento se divide en directo e indirecto. Entre esas exenciones se encuentra el financiamiento público, identificado comúnmente como financiamiento público directo. En este sentido, Aparicio y Pérez (2007) entienden por financiamiento público "el empleo del dinero público en beneficio de los partidos" (p.3).

La enunciación anterior hace notar que existe una relación compleja entre dinero y democracia. Se podría considerar determinante que el dinero invertido en los partidos políticos convierte en realidad a un Estado democrático. Si bien, los fondos públicos destinados a los partidos se pueden significar como el 
combustible que hará funcionar la maquinaria democrática, se hace necesario implementar distintos modelos de administración, basados en modelos matemáticos, para que esta maquinaria cumpla funcionalmente la tarea para la que ha sido dispuesta.

Es indudable que los países han implementado distintos modelos, con diferentes virtudes y defectos, para asignar adecuadamente el financiamiento que proviene de las arcas públicas y han optado, por diversas circunstancias, por los sistemas que combinan el financiamiento público con el privado, es decir, los sistemas mixtos. Gutiérrez y Zovatto (2011), observan que en la actualidad, más partidos políticos participan en elecciones, $\mathrm{y}$ que las formas de financiamiento son más competitivas y especializadas. Aunque no se aclara, es probable que los tipos de financiamiento, su combinatoria o evolución, tengan algún tipo de incidencia en la práctica, pero ello no se ha revelado del todo. Sin embargo, sí se evidencia que mientras los sistemas de financiamiento se especialicen, los resultados se hacen más tangibles.

Por ejemplo, para las elecciones

nacionales de Uruguay en 2014, el financiamiento fue mixto (Pérez y Piñeiro, 2016); un caso similar, según un estudio realizado por el Instituto de Investigaciones Jurídicas de la UNAM y la Organización de los Estados Americanos en 2011, refiere que Chile tiene un financiamiento mixto, el cual está establecido en la Constitución (Fuentes, 2011).

\subsection{Financiamiento de partidos políticos}

Oppo (1976), comenta que la figura de los partidos políticos se origina hacia la primera mitad del siglo XIX, en Europa y Estados Unidos, lugares en los que, al afirmarse el poder de la clase burguesa, se adopta primero la democracia representativa y las instituciones parlamentarias para consolidarla.

Asimismo, en Inglaterra, Oppo (1976) dice que los partidos políticos hacen su aparición con la Reforma Constitucional de 1832 que, ampliando el sufragio, permitió que los estratos industriales y comerciales del país participaran junto a la aristocracia en la gestión de los negocios públicos. Antes de esa fecha, no puede hablarse en Inglaterra de partidos políticos propiamente dichos: Los dos grandes partidos de la aristocracia, surgidos en el siglo XVIII y presentes desde entonces en el parlamento, no tenían fuera del mismo ninguna relevancia y ningún tipo de organización; se trataba de simples etiquetas detrás de las cuales estaban los representantes de un estrato homogéneo no dividido por conflictos de interés.

En la historia, los partidos políticos han desempeñado funciones que permiten dar mayor validez a los procesos democráticos, dan pie a la pluralidad y posibilitan la transformación continua del poder, son de suma importancia en todo Estado democrático en el que los procesos electorales son fundamentales. Sin embargo, no siempre ha existido la regulación del derecho a financiamiento de los partidos políticos (Gómez, 1996; López y López, 2006).

En México, el financiamiento político-legal se autoriza en la Constitución Política de los Estados Unidos Mexicanos (CPEUM) (Congreso de la República de México, 2020). Este financiamiento público a los partidos políticos inicia en 1977 con la constitucionalización de éstos. En la literatura no se cuestiona en general el grado de inequidad establecido en la fórmula para distribuir los fondos. La desigualdad se presenta en la forma de determinar los partidos que tienen derecho al reparto de los recursos disponibles, es decir, con la barrera de entrada, que para el caso de México actualmente es del 3\% de la Votación Total Emitida según la Ley General de Partidos Políticos de 2014 (Congreso de los Estados Unidos Mexicanos, 2014). Una vez obtenido el porcentaje citado, queda patente la regla general para distribuir los recursos a los partidos políticos, Castro (2014) la describe de la siguiente manera:

Un procedimiento de distribución entre

los distintos partidos políticos a través 
Arredondo, Verónica; Vega Esparza, Reina Margarita; Villegas Santillán, María Teresa y Álvarez Diez, Rubén Carlos

Nueva propuesta de financiamiento público a partidos políticos en México

del cual, una parte minoritaria del dinero $(30 \%)$ se distribuye de manera igualitaria entre todos, y la otra mayoritaria $(70 \%)$ de forma desigual, en proporción al peso de los votos obtenidos en la última elección de diputados federales. (p.32)

El Instituto Belisario Domínguez (IBD), institución que depende directamente de la Cámara Alta en México, afirma que "las fórmulas de cálculo producen una importante desigualdad entre partidos políticos de nueva creación y partidos consolidados que compiten en elecciones" (IBD, 2017, p.32).

Ugalde (2017), propone las siguientes vías para reducir el financiamiento público a los partidos políticos nacionales: Cambiar la fórmula de financiamiento para actividades ordinarias de los partidos del $65 \%$ de la Unidad de Medida y Actualización (UMA) al 40\%; utilizar en la fórmula la lista nominal del padrón electoral, compuesta por quienes efectivamente cuentan con credencial para votar; emplear el número de votantes de la última elección en lugar del total de empadronados.

En este sentido, el financiamiento público a los partidos políticos es un privilegio que el Estado mexicano entrega en efectivo. No es un gasto, sino una inversión que favorece el interés público mediante el incremento en la oferta de un servicio público, necesario para fortalecer la democracia.

\subsection{Reformas en el Sistema Electoral de México}

Las reformas electorales hechas a la Constitución Política de los Estados Unidos Mexicanos (CPEUM), encuadran la historia del financiamiento público recibido por los partidos nacionales durante más de 30 años. En este trabajo de investigación se analiza el tema del financiamiento público a los partidos políticos, que los legisladores introdujeron en las siete reformas electorales del sistema político mexicano entre 1977 y 2020. El análisis se concentró particularmente en las reformas aplicadas del 2008 al 2020; las cuales fueron promulgadas de los años 2007 al 2014. Las reformas legisladas en ese periodo corresponden a los sexenios que se indican en la Tabla 1.

Tabla 1

Reformas electorales 2007-2014

\begin{tabular}{ccc}
\hline Reforma & Sexenio & Presidente \\
\hline 1977 & $1976-1982$ & José López Portillo \\
1987 & $1982-1988$ & Miguel de la Madrid Hurtado \\
1990 & $1988-1994$ & Carlos Salinas de Gortari \\
1993 & $1988-1994$ & Carlos Salinas de Gortari \\
1996 & $1994-2000$ & Ernesto Zedillo Ponce de León \\
2007 & $2006-2012$ & Felipe de Jesús Calderón Hinojosa \\
2014 & $2012-2018$ & Enrique Peña Nieto \\
\hline
\end{tabular}

Fuente: Elaboración propia, 2020.

Los presidentes publicaron dichas reformas electorales en el Diario Oficial de la Federación, en cuyo análisis se utiliza el concepto de modelo, que el Diccionario de la Lengua Española de la Real Academia Española (RAE, 2020), define como “el 
esquema teórico de un sistema, o de una realidad compleja, expresado generalmente en forma matemática". Dichos modelos, para el caso de México según la Ley General de Partidos Políticos de 2014 (Congreso de los Estados Unidos Mexicanos, 2014), se integran con dos factores: El procedimiento correspondiente para estimar el monto total a distribuir entre todos los partidos políticos; $\mathrm{y}$, la fórmula para calcular el monto del financiamiento público a distribuir a cada uno de estos, de acuerdo con los votos obtenidos.

El conjunto de las reformas electorales en estudio, se divide en dos grupos. Comparativamente, se advierte que el primero, se enfoca en el financiamiento público destinado a captar la máxima cantidad de votos en las elecciones federales; y el segundo, básicamente, en el financiamiento de las actividades ordinarias de los partidos políticos.

Según la reforma de 1977 de la Constitución Política de los Estados Unidos Mexicanos (CPEUM) referente al Sistema Electoral que deriva en la Ley Federal de Organizaciones Políticas y Procesos Electorales 1977 (Congreso de los Estados Unidos Mexicanos, 1977), fija esa tendencia al determinar que los partidos contarían, en forma equitativa, con un mínimo de elementos para captar votos en elecciones federales. Sin embargo, la reforma de 1987 en el Código Federal Electoral 1987 (Congreso de los Estados Unidos Mexicanos, 1987), no precisa en qué tipo de actividades se invertirían los recursos. Se infiere que se aplicarían al objetivo de acrecentar la votación de los partidos en los procesos electorales, porque las variables para calcular el monto del financiamiento lo sugieren: El costo mínimo de una campaña para diputado, el número de candidatos a diputados de mayoría relativa, el número de votos válidos, la votación efectiva para determinar el importe unitario por voto, entre otras.

Las reformas electorales de 1990: Código Federal de Instituciones y Procedimientos Electorales de 1990 (Congreso de los Estados Unidos Mexicanos, 1990), y de 1993: Código Federal de Instituciones y
Procedimientos Electorales de 1993 (Congreso de los Estados Unidos Mexicanos, 1993), manifiestan expresamente financiamiento en las siguientes vertientes: a) Por financiamiento de la actividad electoral, en primer lugar; y b) Por actividades generales como entidades de interés público, en segundo lugar. En ese orden se incluyen: c) Por subrogación del Estado de las contribuciones que los legisladores habrían de aportar para el sostenimiento de sus partidos; d) Por actividades específicas como entidades de interés público; $y, e$ ) Para el desarrollo de los partidos políticos.

En sentido opuesto, la reforma electoral de 1996: Código Federal de Instituciones y Procedimientos Electorales de 1996 (Congreso de los Estados Unidos Mexicanos, 1996), opta por la alternativa de asignar recursos en efectivo bajo el siguiente orden: a) Para el sostenimiento de actividades ordinarias permanentes; b) Para gastos de campaña y, c) Para actividades específicas como entidades de interés público.

La reforma electoral 2007 que se exhibe en el Código Federal de Instituciones y Procedimientos Electorales de 2008 (Congreso de los Estados Unidos Mexicanos, 2008), mantiene la tendencia de aplicar el financiamiento público referido en la de 1996: a) Para el sostenimiento de actividades ordinarias permanentes; b) Para gastos de campaña; y, c) Por actividades específicas como entidades de interés público. La reforma electoral de 2014 a través de la Ley General de Partidos Políticos (Congreso de los Estados Unidos Mexicanos, 2014), dispone el siguiente orden: a) Para financiar las actividades ordinarias permanentes; b) Para gastos de Campaña; y, c) Por actividades específicas como entidades de interés público.

La cantidad de variables que las reformas electorales de 1987, 1990, 1993 y 1996 emplearon para calcular el monto de actividades a financiar, apuntalaron un incremento de los recursos otorgados. Con base en la Ley Federal de Organizaciones Políticas y Procesos Electorales que conllevo al Código Federal Electoral 1987 (Congreso de los Estados Unidos Mexicanos, 1987), 
Arredondo, Verónica; Vega Esparza, Reina Margarita; Villegas Santillán, María Teresa y Álvarez Diez, Rubén Carlos

Nueva propuesta de financiamiento público a partidos politicos en México

el modelo de reforma electoral de 1987, utilizó las variables de costo mínimo de una campaña electoral para diputado y número de candidatos a diputados de mayoría relativa. Según el Código Federal de Instituciones y Procedimientos Electorales de 1990 (Congreso de los Estados Unidos Mexicanos, 1990) y el Código Federal de Instituciones y Procedimientos Electorales de 1993 (Congreso de los Estados Unidos Mexicanos, 1993), los modelos de 1990 y 1993, aplicaron las variables de costo mínimo de una campaña para diputado y una para senador, número de candidatos propietarios a diputados de mayoría relativa y número de candidatos propietarios a senadores.

El modelo electoral de 1996 operaba con las variables: Costos mínimos de una campaña para diputado, una para senador y otra para presidente, número de diputados a elegir, y de partidos políticos con representación en las Cámaras del Congreso. Las reformas electorales de los años 2007 y 2014 introdujeron un modelo de financiamiento que, en teoría, disminuiría la cantidad de recursos públicos que se otorgaban a los partidos políticos.

Reyes (2007), estimó que el ahorro que se generaría en las finanzas públicas por la reducción del financiamiento público derivado de la reforma, en materia de la fórmula de financiamiento público para los partidos, sería de dos mil 542 millones de pesos, durante el periodo 2008-2009. Las variables correspondientes a la fórmula del modelo son: El padrón electoral y el salario mínimo diario vigente para el Distrito Federal, reemplazado el 29 de febrero de 2016 por la Unidad de Medida y Actualización (UMA) que publica diariamente el Banco de México.

Por otra parte, de acuerdo con Hernández y Chumaceiro (2018) la democracia "ha sido un proceso de definición tanto coyuntural como estructural, caracterizada por las perspectivas ideológicas del momento, determinada por elites, por grupos de interés corporatizados en función de la representatividad y/o participación y de los estatus quo dominantes" (p.57). Asimismo, se sabe que aunque existen diversos modelos de democracia, algunas veces no es posible alcanzar con ellos los mecanismos para generar la participación ciudadana que puedan ser aplicables en el entorno en el que se desarrollan los regímenes democráticos (Contreras y Montecinos, 2019); sin embargo, debido a que los partidos políticos nacionales han sido constitucionalmente entidades de interés público, el tema de su financiamiento no es coyuntural. Por ello, el debate social y político persistirá en el mediano y largo plazo.

Por ese motivo, es importante considerar que la disponibilidad de modelos para analizar las variables que determinan el adecuado monto y reparto de financiamiento a los partidos - con base en distintos criterios para maximizar la equidad entre los que participan en los procesos electorales - es limitada. Esta situación no garantiza la existencia de un sistema democrático moderno.

En concreto, las instituciones competentes que se encargan de organizar, arbitrar y vigilar las contiendas electorales, carecen de una metodología equitativa o justa, por decirlo de un modo más sencillo, hay una falta de metodología para la asignación de los recursos económicos a partidos políticos, considerando variables significativas que garanticen la transparencia de los recursos otorgados, de tal forma que dé un trato igualitario a los partidos políticos.

Debido a la falta de un modelo de financiamiento público a los partidos políticos, este estudio se enfoca en desarrollar una propuesta orientada a generar un mayor ahorro en el erario público, así como una mayor equidad en el trato a dichos partidos, con base en la proporcionalidad entre el porcentaje de votos y el de recursos otorgados a los mismos.

\section{Metodología}

Como se comentó en la sección anterior, el objetivo principal de este estudio fue proponer un modelo que permita estimar el recurso que debe otorgarse a cada partido político, evitando por un lado, el trato inequitativo a los partidos políticos y por el 
otro, estimar el recurso global con base en la tasa de participación electoral; tomando en cuenta únicamente el número de votos recibidos por cada uno de los partidos, siempre y cuando estos hayan superado la barrera a la entrada en el reparto de escaños.

Se propuso una función continua para la estimación de la barrera a la entrada, con el fin de determinar qué partidos políticos tienen derecho al reparto de recursos financieros. Dicha función depende del número de votos que cada partido político obtuvo a nivel nacional para elegir diputados federales de representación proporcional, y la resta de un $3 \%$ de la Votación Total Emitida, a cada uno de los partidos políticos que han sido parte de la contienda electoral en cuestión. En ese sentido, se analiza el recurso asignado a los partidos políticos en el periodo de 2008 a 2020. Se contempló este periodo de tiempo pues es aquel en el que se aplicó la ley vigente para tal efecto. Finalmente, se estimó un modelo que permite dar un pronóstico de la estimación de electores que ejercerán su derecho a voto; para este caso se utilizará un modelo de Promedios Móviles Ponderado.

La Votación Total Emitida (VTE), vista a través del tiempo ha tenido un comportamiento similar, no ha presentado grandes cambios en las distintas elecciones efectuadas en México. Así, desde el punto de vista de las series de tiempo la VTE es una variable que presenta una serie de tiempo estable. El modelo de series de tiempo de Promedios Móviles Ponderados es propio para datos que tienen el comportamiento de series de tiempo estables, sin grandes fluctuaciones o cambios abruptos en las observaciones (Hanke y Reitsh, 1996). Es por ello, que se sugiere el uso del modelo citado para la estimación de la VTE.

\section{Resultados}

En este apartado se muestran los resultados de las metodologías y procedimientos propuestos, los cuales se aplicaron a datos reales para el caso de México a nivel federal, con la finalidad de mostrar una nueva metodología para el financiamiento público a los partidos políticos, desarrollado con base en la proporcionalidad entre el porcentaje de votos y el de recursos otorgados a los partidos, el reparto equitativo del monto calculado, y de sus efectos en la competencia electoral; además de considerar como barrera a la entrada, una función continua que evite la inequidad entre los recursos otorgados a los partidos políticos. Asimismo, se aplicará la nueva propuesta y se presentarán los posibles ahorros monetarios.

\subsection{Nueva propuesta de metodología para la asignación de recursos públicos a los partidos políticos en México}

Esta nueva propuesta contempla en primer lugar, el procedimiento para estimar la Votación Total Emitida, considerada como la participación electoral. Enseguida, un procedimiento para la barrera a la entrada de reparto de recursos públicos. Finalmente, se presenta la nueva propuesta de asignación de presupuesto para financiamiento público de partidos políticos.

\section{a. Procedimiento propuesto para estimar la participación electoral (Votación Total Emitida, VTE)}

Para estimar la tasa de participación electoral y/o votación total, se utilizó el modelo de series de tiempo conocido como Promedios Móviles Ponderados. Se consideró estimar la votación total emitida (VTE) mediante este método, dado que es adecuado para datos que no registran variaciones abruptas en el tiempo, es decir, el número total de electores tuvo un comportamiento que se ha mantenido estable (Hanke y Reitsh, 1996), en términos del porcentaje de electores que emiten el voto. En este caso la VTE tiene un comportamiento de esta forma.

Además, este método le asignó a cada observación una importancia diferenciada a 
Arredondo, Verónica; Vega Esparza, Reina Margarita; Villegas Santillán, María Teresa y Álvarez Diez, Rubén Carlos

Nueva propuesta de financiamiento público a partidos politicos en México

cada uno de los datos que componen dicho promedio, es decir, en el promedio móvil ponderado se asignan pesos a cualquier dato del promedio (siempre que la sumatoria de las ponderaciones sean equivalentes al 100\%). Entre mayor sea el peso de la observación, mayor será la importancia dada a la misma. Así, este método permite dar especial relevancia a la elección inmediata anterior.

El modelo de Promedios Móviles, considera un número constante de datos para hacer la estimación, una vez que se tiene nueva información, de observaciones nuevas de la variable en cuestión; los valores más antiguos son sustituidos y ponderados en la medida determinada a priori. La fórmula matemática de este modelo es:

$$
\mathrm{VTE}_{\text {Estimada }(t+1)}=\sum_{\mathrm{t}=1}^{\mathrm{n}} \mathrm{C}_{\mathrm{t}} \mathrm{VTE}_{\mathrm{t}}
$$

Dónde:

VTE $_{\text {Estimada }(t+1)}$ : Votación total emitida estimada en el periodo $t+1$

$\mathrm{C}_{\mathrm{t}}$ : Ponderación de la VTE en el periodo $\mathrm{t}$

$\mathrm{n}$ : Número de periodos considerados

Además, la sumatoria:

$$
\sum_{\mathrm{r}=1}^{\mathrm{n}} \mathrm{C}_{\mathrm{t}}=100 \%
$$

Se estimó el promedio de las últimas tres observaciones dando un mayor peso al último dato, es decir, se tiene en cuenta que la elección más próxima anterior tiene especial importancia.

\section{b. Procedimiento propuesto para determinar la barrera a la entrada de reparto de recursos públicos}

La propuesta disminuye la inequidad entre partidos políticos que resulta de las barreras electorales que se aplican comúnmente (partido político o coalición que no satisface un porcentaje específico de votos, es excluido del reparto de escaños de representación proporcional y por tanto, del reparto de recursos públicos) se presenta en Ramírez (2013), quienes han propuesto utilizar una barrera a la entrada de reparto de escaños que sea una función continua (que depende del número de votos) y no como una función discontinua. El salto o discontinuidad se da exactamente en el valor de la barrera a la entrada.

Como se ha comentado, actualmente los partidos políticos que no reciben el $3 \%$ o más de votos respecto de la Votación Total Emitida (VTE), no tienen derecho a reparto de recurso público (y de escaños por representación proporcional). De esta manera, si el partido A obtiene $2.99 \overline{9} \%$ de la VTE, no recibe financiamiento. Por otra parte, si un partido político $\mathrm{B}$, cuenta con un solo voto más de los que obtiene el partido A, y alcanza el 3\% de la VTE, este partido B obtiene el 3\% del total de los recursos disponibles.

Lo anterior, implica que la función de asignación de recursos actualmente presenta una discontinuidad en el valor de la barrera a la entrada. Además, otra desventaja de esta discontinuidad generada este tipo de barrera, es que contribuye a la presencia de desproporcionalidad electoral, es decir, diferencias entre el porcentaje de votos y el de escaños recibidos por los partidos políticos participantes (Martínez-Panero, et al., 2019). Para este caso se propone restar el 3\% de los votos a todos los partidos políticos, y a partir de los votos restantes, se otorgará la proporción de recursos financieros que corresponda. Así habrá trato igual para todos los partidos políticos, sin importar si son grandes o pequeños (en términos de votos).

Formalmente, lo anterior es: A los partidos se les disminuye la misma cantidad que representa el porcentaje $r$ (barrera a la entrada) de la votación total emitida. Así para cada partido $i$ la cantidad de votos que se toma en cuenta para el reparto de recursos públicos será:

$$
V_{i}^{\prime}=V_{i}-r * V
$$

Este será un mínimo número de votos que deberá tener el partido para acceder a los fondos públicos de financiamiento. Aquí se ha 
formulado y adecuado la función del umbral de partidos con derecho al reparto de escaños por representación proporcional, contemplada por Ramírez (2013). Dicha función del umbral de exclusión, evita, por ejemplo, el caso extremo en el que por diferencia de un voto un partido político recibe porcentaje de escaños fijo (el 3\% para el caso de México) $\mathrm{y}$ otro partido con un voto menos obtenga cero escaños. A partir de lo anterior, se hace la siguiente propuesta para el reparto de recursos por partidos políticos:

Sea $V^{\prime}=\sum_{i=1}^{n} V_{i}^{\prime}, \quad V_{i}^{\prime}=V_{i}-r * V T E$. Tomando en cuenta el actual sistema electoral de México de acuerdo con la Ley General de Partidos Políticos de 2014 (Congreso de los Estados Unidos Mexicanos, 2014) y la nueva propuesta de umbral de exclusión, el monto $F_{i}^{\prime}$ de financiamiento a cada partido político será dada por la siguiente función:

$$
F_{i}^{\prime}=\left\{\begin{array}{lll}
0 & \text { si } & V_{i}^{\prime}<r * V T E \\
F * \frac{V_{i}^{\prime}:}{V T E} & \text { si } & V_{i}^{\prime} \geq r * V T E
\end{array}\right.
$$

\section{Dónde:}

$V T E$ : Es la votación total emitida de acuerdo a la Ley General de Partidos Políticos (2014) (participación electoral).

$V_{i}^{\prime}$ : Porcentaje de votos considerados para el reparto de recursos públicos (una vez que se ha restado la cantidad de votos $r * V T E$ ).

$F$ : Financiamiento total a repartir entre los partidos políticos que tengan derecho.

Es de hacer notar que, aquí solo se está esquematizando la función general del reparto del financiamiento público, es decir, solo se establece qué partidos tendrían derecho al mismo y cuáles no. La cantidad exacta del monto se determinará según indique la fórmula que se establezca para este fin.

\section{c. Nueva propuesta para asignación de presupuesto para financiamiento público de partidos políticos}

Para la determinación de qué partidos políticos tienen derecho a la obtención de recursos públicos, de acuerdo a lo que establece la ley, se considera el límite (barrera a la entrada) de votos que deben pasar los partidos para tal fin. Para ello, se estimó el número de votos $-\mathrm{VTE}_{\text {Estimada }(t+1)^{-}}$que se utiliza para el cálculo de la cantidad total de los recursos económicos que se distribuiría entre todos los partidos que tuvieran derecho a ello. Como ya se expuso, este procedimiento es una nueva propuesta que contempla el modelo Promedios Móviles Ponderados.

Una vez que se obtuvieron los resultados de los dos puntos anteriores, se aplicó el procedimiento indicado por la Ley General de Partidos Políticos 2014 (Congreso de los Estados Unidos Mexicanos, 2014), excepto que en lugar de usar el número total de ciudadanos inscritos en el padrón electoral federal para los partidos nacionales, o local para los partidos locales, según sea el caso, a la fecha de corte de iulio de cada año, se utiliza la $\mathrm{VTE}_{\text {Estimada }(t+1)}$ propuesta en este trabajo. El procedimiento indicado por la Ley General de Partidos Políticos del año 2014 (Congreso de los Estados Unidos Mexicanos, 2014) es:

a. El número total de ciudadanos inscritos en el Padrón Electoral Federal para los partidos nacionales, o local para los partidos locales, según sea el caso, a la fecha de corte de julio de cada año.

b. El monto del salario mínimo vigente para el Distrito Federal para los partidos políticos nacionales, o el salario mínimo de la región en la cual se encuentre la entidad federativa, para el caso de los partidos políticos locales.

c. Conociendo el valor de ambas variables, lo que sigue es multiplicar el número total de los inscritos en el padrón por el $65 \%$ del monto del salario mínimo.

d. El resultado de la operación señalada en el inciso anterior constituye el financiamiento público anual a los partidos políticos por sus actividades ordinarias permanentes.

e. El $30 \%$ de la cantidad que resulte de acuerdo a lo señalado anteriormente, se distribuirá entre los partidos políticos en forma igualitaria y el $70 \%$ restante de acuerdo con 
Arredondo, Verónica; Vega Esparza, Reina Margarita; Villegas Santillán, María Teresa y Álvarez Diez, Rubén Carlos

Nueva propuesta de financiamiento público a partidos politicos en México

el porcentaje de votos que hubieren obtenido en la elección de diputados inmediata anterior.

f. En el año de la elección en que se renueven el Poder Ejecutivo Federal o local y las dos Cámaras del Congreso de la Unión o el Congreso de alguna entidad federativa, a cada partido político nacional o local, en su caso, se le otorgará para gastos de campaña un monto equivalente al $50 \%$ del financiamiento público que para el sostenimiento de sus actividades ordinarias permanentes le corresponda en ese año.

g. En el año de la elección en que se renueve solamente la Cámara de Diputados federal o los Congresos de las entidades federativas, a cada partido político nacional o local, respectivamente, se le otorgará para gastos de campaña un monto equivalente al $30 \%$ del financiamiento público que para el sostenimiento de sus actividades ordinarias permanentes le corresponda en ese año.

\subsection{Resultados de la implementación de la nueva metodología para la asignación de recursos públicos a los partidos políticos en México}

En este apartado, se analizó el recurso asignado a los partidos políticos en el periodo de 2008 a 2020. Se contempló este periodo de tiempo pues es aquel en el que se aplicó la ley vigente para tal efecto. La tasa de participación del voto fue alrededor del $55 \%$ en dicho periodo, en este sentido, se otorgó en términos generales a los partidos políticos un $45 \%$ más de los recursos que realmente deberían recibir considerando su número de votos.

Por otra parte, una vez establecido el monto total de los recursos públicos asignados a los partidos políticos, existieron variaciones entre la proporción de recursos públicos que recibieron y la proporción de votos que obtuvieron. Esto debido a que se reparte el $100 \%$ de los recursos disponibles entre los partidos que tienen derecho a ello, es decir, aquellos que pasan la barrera a la entrada. Con base en lo anterior, se sabe que se hace una distribución de los recursos totales a aquellos partidos que se encuentran en ese supuesto, sin cuantificar los votos nulos y votos obtenidos por los candidatos independientes.

Si se efectúa una comparación entre el presupuesto otorgado actualmente a los partidos políticos en México y el asignado mediante la metodología propuesta en este trabajo, se evidencian diferencias significativas. En la primera, cada partido depende en gran medida de la cantidad de votos que obtiene, así como de una Votación Total Estimada; en la segunda, se contempla el número real de electores, los cuales en su mayoría, no acuden a las urnas.

En México, una parte del financiamiento público $(70 \%)$, se reparte de manera proporcional a los partidos políticos según el porcentaje de votos de acuerdo con la Votación Válida Emitida (Votación Total Emitida menos los votos recibidos por los candidatos(as) independientes y los nulos), y la otra, se reparte de forma equitativa $(30 \%)$.

Con la finalidad de exponer la nueva metodología propuesta en este artículo, se explican y analizan las cifras que se obtuvieron para el financiamiento, para lo cual se tomó en cuenta el año en el que se dio el proceso electoral más reciente (2018); en las estimaciones para 2018, se retoma la votación del año 2015.

Cabe resaltar el hecho de que el Partido del Trabajo estuvo en el límite de la votación que no le permitió recibir recursos públicos, con un $2,9 \%$ de la Votación Total Emitida, así cualquier partido que haya obtenido el $3 \%$ o poco más de esta votación, recibió una cantidad importante de dinero con una diferencia de menos del $0,1 \%$, esta inequidad no sucedería con la función propuesta para la barrera a la entrada en el reparto de recursos disponibles para los partidos políticos.

Los resultados de la aplicación de la propuesta y su comparación con los montos obtenidos bajo las leyes aplicables para el periodo de tiempo contemplado en este trabajo, se concentran en la Tabla 2. En la columna A, el monto real asignado a los partidos políticos; en la columna B, el monto que corresponde a la estimación considerando la propuesta presentada, el ahorro que se habría derivado 
en pesos mexicanos; y, la última columna, presenta el ahorro en porcentaje.

Tabla 2

Estimación del ahorro en financiamiento a partidos 2008-2020

\begin{tabular}{lcccc}
\hline AÑO & A: Real Asignado & B: Propuesta & Ahorro (A-B ) & \% ahorro \\
\hline 2020 & $\$ 5.239 .001 .651$ & $\$ 2.908 .540 .636$ & $\$ 2.894 .629 .285$ & 44,4 \\
2019 & $\$ 4.870 .560 .864$ & $\$ 2.774 .628 .657$ & $\$ 2.095 .932 .207$ & 43,0 \\
2018 & 6.444 .499 .869 & 3.044 .503 .702 & 3.399 .996 .167 & 52,8 \\
2017 & 4.059 .213 .905 & 1.983 .049 .886 & 2.076 .164 .019 & 51,2 \\
2016 & 3.953 .658 .321 & 1.903 .228 .327 & 2.050 .429 .994 & 51,9 \\
2015 & 5.199 .695 .918 & 3.138 .313 .770 & 2.061 .382 .148 & 39,6 \\
2014 & 3.925 .109 .677 & 2.028 .031 .361 & 1.897 .078 .316 & 48,3 \\
2013 & 3.670 .843 .753 & 1.951 .780 .517 & 1.719 .063 .236 & 46,8 \\
2012 & 5.142 .514 .888 & 2.126 .506 .024 & 3.016 .008 .864 & 58,7 \\
2011 & 3.212 .932 .809 & 1.373 .920 .783 & 1.839 .012 .026 & 57,2 \\
2010 & 2.997 .358 .834 & 1.319 .717 .288 & 1.677 .641 .546 & 56,0 \\
2009 & 3.633 .067 .352 & 2.042 .636 .216 & 1.590 .431 .136 & 43,8 \\
2008 & $\$ 2.690 .888 .670$ & $\$ 1.319 .717 .288$ & $\$ 1.371 .171 .382$ & 51,0 \\
\hline
\end{tabular}

Fuente: Elaboración propia, 2020.

Para el financiamiento público a los partidos políticos de 2019, la votación nacional emitida que se tomó como referencia fue de 40.7 millones de votos. Esta votación correspondiente a las elecciones previas, que beneficiaron enmayormedidaalPartidoMorena (Morena), al Partido Acción Nacional (PAN) y al Partido de la Revolucionario Institucional (PRI), los cuales concentraron 79,5\% del total de votos emitidos. El financiamiento público anual para el sostenimiento de sus actividades ordinarias permanentes (SAOP), que resulta de multiplicar el $65 \%$ de la UMA por el padrón electoral total en ese año $\left(90^{\prime} 259,589\right)$, fue de 4.728,7 millones de pesos. De acuerdo con lo expuesto en los apartados anteriores, el $70 \%$ de dicho monto (3.310,1 millones de pesos) se distribuyó entre los partidos políticos según el porcentaje de la votación total emitida alcanzada, mientras que el $30 \%$ restante (1.418,6 millones de pesos) se repartió de forma igualitaria.

A lo anterior se debe sumar un monto equivalente al $50 \%$ del financiamiento público anual para el SAOP que se distribuye igualmente según la participación de cada partido en la votación, lo que da como resultado el financiamiento total a los partidos. De esta manera, en 2019 Morena, el PAN y el PRI concentraron 3.339,5 millones de pesos del presupuesto que se destinó a los partidos en ese año; en sentido opuesto, los partidos que menos recursos recibieron fueron el Partido del Trabajo (PT) y Movimiento Ciudadano (MC).

Cabe señalar que el monto disponible en la propuesta es muy difícil que sea igual al monto que finalmente se otorga a los partidos 
Arredondo, Verónica; Vega Esparza, Reina Margarita; Villegas Santillán, María Teresa y Álvarez Diez, Rubén Carlos

Nueva propuesta de financiamiento público a partidos politicos en México

políticos. La razón, como se ha dicho, es que para estimar el monto disponible se emplea el método de Promedios Móviles Ponderados, utilizando las últimas tres elecciones para diputados locales. En dicha estimación, se toma en cuenta los votos nulos, los votos de partidos políticos que no han pasado la barrera a la entrada y los candidatos independientes, si es pertinente. En la propuesta, a diferencia de la ley vigente para asignación de recursos públicos a partidos políticos, este recurso no se redistribuyó entre los partidos que sí pasaron la barrera a la entrada y la propuesta es que se regrese el recurso a la federación.

Así, por ejemplo para el año 2019, se podría disponer de un monto de $\$ 2.774 .628 .657,00$ pero solo se distribuiría un monto de $\$ 2.236 .435 .232$ (Ver Tabla 2) pues los candidatos no registrados, votos nulos $\mathrm{y}$ candidatos independientes, no obtienen recursos aún si hubiesen pasado la barrera a la entrada de financiamiento.

En ese sentido, es posible apreciar que considerar un promedio móvil sobre la votación nacional emitida, implica una base menor para calcular el financiamiento para los partidos políticos, con diferencias porcentuales que van desde $39,6 \%$ para 2015 hasta $59,7 \%$ en 2012 ; de esta manera, el financiamiento propuesto implica en promedio un gasto público menor en $50,1 \%$ para el periodo $2008-2020$.

Además, las diferencias entre el porcentaje de votos y el de recursos obtenidos por los partidos políticos a través de la propuesta, son notablemente menores. Cabe señalar, que estas diferencias se deben principalmente a que en la propuesta a todos los partidos políticos (no solo a los que obtuvieron 3\% o menos de la VTE) se les resta una cantidad fija de votos (el 3\% de la VTE, en este caso).

\section{Conclusiones}

El tema del reparto de los recursos financieros a los partidos políticos siempre será un fenómeno que genere polémicas $\mathrm{y}$ disyuntivas tanto en las instituciones participantes como entre los ciudadanos. Para salvar esta controversia es necesario presentar de una manera transparente una propuesta metodológica clara y concisa, además de justa y bien argumentada, sobre la asignación de recursos a los partidos políticos dependiendo de las variables bajo las que se han construido como instancias políticas y del desarrollo de un proceso de competencia electoral.

El sistema actual de administración ignora variables importantes en la repartición de recursos, factores que pueden preverse cualitativa y cuantitativamente si se recurriera al estamento científico. Por ejemplo, una barrera a la entrada que genere trato equitativo a los partidos políticos. Probablemente esto no sería relevante si no fuese porque el patrocinio de los partidos políticos corre a cargo de los recursos públicos, es decir, fondos que tienen su origen en el dinero de los contribuyentes. Este es un motivo trascendental por lo que el sistema debería ser evaluado y modificado por las instancias pertinentes.

Actualmente, las competencias electorales se encuentran bajo el foco de la desconfianza por parte de los ciudadanos, principalmente porque los partidos políticos no solo rebasan casi siempre los topes de campaña, sino porque considerando los presupuestos asignados a distintos rubros, como por ejemplo, los apoyos al campo, el financiamiento recibido por los partidos políticos parece ser excesivo. Cabe señalar que para 2018, periodo de elecciones, se otorgó $6.573,4$ millones de pesos. Este presupuesto es casi el doble de lo que se presupuesta en educación para grupos vulnerables y casi igual a lo que se les asigna en salud en todo el país; y representa también casi el triple de lo que se presupuestó para el desarrollo rural en todo México en el año 2019.

Como se ha expuesto, los ahorros que se generarían de aplicar la propuesta de financiamiento público a los partidos políticos, es de un poco más del $50 \%$ de lo que actualmente reciben, con lo que evidentemente, podrían verse beneficiadas instancias de desarrollo social y económico desprotegidos en México. 
Resulta trascendental no solo transparentar el gasto y los recursos de los partidos políticos, sino tener un método más adecuado en la administración de los recursos que se les asignan. El ahorro que es posible obtener en su financiamiento podría ser trasladado a rubros como la educación y la salud, temas que en esta coyuntura se han visto fuertemente afectados, lo que es bien sabido necesitan ser apuntalados para que, como nación y sociedad, se pueda responder ante situaciones no previstas, por ejemplo la pandemia del COVID-19. Podría decirse que el gasto en mejorar una democracia nunca es excesivo, si el último fin es evolucionar para conseguirla o mantenerla. Sin embargo, es posible plantearse más de una solución para lograrlo de una manera más económica, en condiciones más igualitarias, justas, legales, que sean de la aprobación de los ciudadanos.

\section{Referencias bibliográficas}

Aparicio, J., y Pérez, J. M. (2007). Financiamiento público a partidos políticos. En Grandes temas para un Observatorio Electoral Ciudadano (Vol. III) (pp. 211-232). Instituto Electoral del Distrito Federal.

Castro, J. T. (2014). Dos fuentes de financiamiento público a los partidos políticos nacionales: Federal y estatal. Espacios Públicos, 17(41), 31-65.

Congreso de la Republica de Mexico (2020). Constitución Política de los Estados Unidos Mexicanos. Congreso de la Unión. https://bit.ly/39SG8ZL

Congreso de los Estados Unidos Mexicanos (1977). Ley Federal de Organizaciones Políticas y Procesos Electorales 1977. Congreso de la Union. https://bit. ly/2LMnuL9

Congreso de los Estados Unidos Mexicanos (1987). Código Federal Electoral 1987. Congreso de la Unión. https:// bit.ly/399ALG1

Congreso de los Estados Unidos Mexicanos
(1990). Código Federal de Instituciones y Procedimientos Electorales 1990. Congreso de la Unión. https://bit.ly/3iOkdah

Congreso de los Estados Unidos Mexicanos (1993). Código Federal de Instituciones y Procedimientos Electorales 1993. Congreso de la Unión. https://bit.ly/3639F1N

Congreso de los Estados Unidos Mexicanos (1996). Código Federal de Instituciones y Procedimientos Electorales 1996. Congreso de la Unión. https://bit.ly/39Ty1vP

Congreso de los Estados Unidos Mexicanos (2008). Código Federal de Instituciones y Procedimientos Electorales 2008. Congreso de la Unión. http://www.diputados.gob.mx/ LeyesBiblio/abro/cofipe/COFIPE abro 14ene08.pdf

Congreso de los Estados Unidos Mexicanos (2014). Ley General de Partidos Politicos 2014. Congreso de la Unión. https://bit.ly/3iDNXq6

Contreras, P., Montecinos, E. (2019). Democracia y Participación Ciudadana Tipología y Mecanismos para la implementación. Revista de Ciencias Sociales, $X X V(2), 178-191$.

Fuentes, C. (2011). Financiación de partidos políticos en Chile. En P. Gutiérrez y D. Zovatto (Coords.), Financiamiento de los partidos políticos en América Latina (pp. 135-183). Universidad Nacional Autónoma de México (UNAM), Instituto de Investigaciones Jurídicas y Organización de los Estados Americanos.

Gómez, G. (1996). Algunas consideraciones sobre el financiamiento público de los partidos políticos. Revista de Ciencia Política, 18(1-2), 115-129.

Gutiérrez, P., y Zovatto, D. (2011). Balance regional: Financiamiento político 
Arredondo, Verónica; Vega Esparza, Reina Margarita; Villegas Santillán, María Teresa y Álvarez Diez, Rubén Carlos

Nueva propuesta de financiamiento público a partidos politicos en México

en América Latina 2004-2010. En P. Gutiérrez y D. Zovatto (Coords.), Financiamiento de los partidos políticos en América Latina (pp. 3-15). Universidad Nacional Autónoma de México (UNAM), Instituto de Investigaciones Jurídicas y Organización de los Estados Americanos.

Hanke, J. E., y Reitsch, A. G. (1996). Pronósticos en los negocios. Prentice Hall Inc.

Hernández, J. J., y Chumaceiro, A. C. (2018). Acercamiento histórico a la participación ciudadana en Venezuela: Modelo de relación Estado-Sociedad (1958-2012). Revista de Ciencias Sociales (Ve), XXIV(2), 56-67.

Instituto Belisario Domínguez (2017). Gasto y fiscalizaciónelectoralen México: 1997 2017. Temas estratégicos 48. http:// www.bibliodigitalibd.senado.gob.mx/ bitstream/handle/123456789/3690/

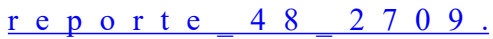
pdf? sequence $=5 \&$ is Allowed $=\mathrm{y}$

López, J., y López, J. (2006). Fuentes del financiamiento para los partidos políticos en México. Espacios Públicos, 9(18), 71-96.

Martínez-Panero, M., Arredondo, V., Peña, T., y Ramírez, V. (2019). A new quota aproach to electoral disproportionality. Economies, 7(1), 1-17. https://doi. org/10.3390/economies 7010017

Ohman, M. (2015). Introducción al financiamiento político. En E. Falguera, S. Jones, y M. Ohman (Eds.), El financiamiento de los partidos politicos y las campañas electorales: Manual sobre financiamiento politico (pp. 1-11). Instituto Internacional para la Democracia y Asistencia Electoral
(IDEA Internacional), Tribunal Electoral del Poder Judicial de la Federación.

Oppo, A. (1976). Partidos políticos. En N. Bobbio, N. Matteucci y G. Pasquino (Eds.), Diccionario de Politica (pp. 23-32). Siglo XXI Editores.

Pérez, V., y Piñeiro, R. (2016). Uruguay 2015: Los desafíos de gobernar por izquierda cuando la economía se contrae. Revista de Ciencia Política, 36(1), 339-363. http://dx.doi.org/10.4067/ $\underline{\mathrm{S} 0718-090 \times 2016000100015}$

Ramírez, V. (Ed.) (2013). Sistema Electoral para el Congreso de los Diputados. Propuesta para un parlamento más ecuánime, representativo $y$ gobernable. Editorial Universidad de Granada.

Real Academia Española - RAE (2020). Diccionario de la lengua española. https://dle.rae.es

Reyes, M. (2007). La reducción del financiamiento público de los partidos políticos nacionales como resultado de la iniciativa de reforma al artículo 41 constitucional. Centro de Documentación, Información y Análisis, Cámara de Diputados.

Ugalde, L. C. (2017). Cinco modalidades para reducir el finaciamiento de los partidos políticos. Integralia Consultores. https://integralia.com.mx/web/ wp-content/uploads/2019/08/5modalidades-para-reducir-elfinanciamiento-a-partidos.pdf

Zovatto, D. (2017). El financiamiento político en América Latina. Studia Politicae, (40), 7-52. 